\title{
Tratamiento de la adicción a la nicotina en una persona fumadora de puros
}

\author{
Míguez, M.C.; BecoñA, E. \\ Universidad de Santiago de Compostela \\ Enviar correspondencia: \\ María del Carmen Míguez. Universidad de Santiago de Compostela. Unidad de Tabaquismo. Facultad de Psicología. Departamento de Psicología Clínica y Psicobiología. \\ Campus Universitario Sur. 15782 Santiago de Compostela.
}

\section{RESUMEN}

Aunque la prevalencia del consumo de cigarros puros es inferior a la de otras formas de consumir tabaco, como los cigarrillos, su consumo también crea adicción y conlleva riesgos para la salud. En este trabajo, se presenta la evaluación y el tratamiento de la conducta de fumar en un varón de 48 años de edad con una historia de 27 años de consumo. Éste asistió a un programa psicológico conductual multicomponente para dejar de fumar de seis sesiones (una por semana). El tratamiento incluyó un contrato motivacional, autorregistro de la conducta de fumar, representación gráfica, información acerca del tabaco, reducción gradual de ingestión de nicotina y alquitrán, control de estímulos, tareas para evitar el síndrome de abstinencia de la nicotina y feedback fisiológico (cantidad de monóxido de carbono en aire espirado).

El sujeto dejó de fumar al final de tratamiento y continuó abstinente en los seguimientos llevados a cabo a 1, 3, 6, 9, 12 y 24 meses después de finalizado el tratamiento.

Palabras clave: puros, dejar de fumar, programa psicológico conductual multicomponente.

\section{SUMMARY}

Althought cigar smoking is less prevalent among smokers, cigar consumption also becomes addictive and is a health hazard. We report in this study on the evaluation and treatment of a cigar smoker -a 48- year old man- with a consumption history of 27 years. The subject attended a multicomponent behavioral psychological programme for smoking cessation in six weekly sessions. The treatment included a motivational contract, self-monitoring and graphical representation of cigarette consumption, information regarding tobacco, nicotine fading, stimulus control procedure, strategies to avoid withdrawal syndrome, and physiological feedback (knowledge of amount of expired air carbon monoxide).

The subject was abstinent at the end of treatment, and at the 1-, 3-, 6-, 9-, 12- and 24-month follow-ups.

Key words: cigars, smoking cessation, multicomponent behavioral psychological programme.

\section{INTRODUCCIÓN}

E problema generado por la conducta de fumar se ha convertido, en las últimas décadas de nuestro siglo, en la primera causa de muerte evitable (USDHHS, 2000).

La introducción y conocimiento del tabaco por parte de nuestra cultura occidental se remonta a 1492, fecha en la que Cristobal Colón realiza su primer viaje de expedición hacia el Nuevo Mundo (Llanos, 1985). La forma de consumir tabaco a lo largo de la historia ha ido cambiando. Hoy en día, se consume principalmente en forma de cigarrillos, puros o tabaco de pipa. Con anterioridad al siglo XVIII, el tabaco se mascaba y se consumía en forma de rapé (en polvo). A partir del siglo XVIII, se pasa a un modo totalmente distinto de fumar: a la pipa. Posteriormente, en el siglo XIX, el tabaco se consume fundamentalmente de dos formas: en cigarro puro y mascado. Y, a partir de la Primera Guerra Mundial se extiende con gran fuerza el uso de los cigarrillos en Europa y en el resto de los países. Su bajo coste y comodidad de consumo propició un incremento vertiginoso del consumo de cigarrillos que, hasta nuestros días, es la forma más habitual de consumir esta sustancia.

El tabaco se elabora a partir de hojas secas de la planta Nicotiana Tabacum. A diferencia de los cigarri- 
llos, los puros, o también denominados cigarros puros, se elaboran enrollando una o varias hojas de tabaco. Así, tanto la envoltura como el contenido están hechos sólo de tabaco, de ahí su denominación de "puros", puesto que otros modos de fumar contienen diversos componentes que imposibilitan la pureza de los mismos.

Aunque la prevalencia de consumo de puros es menor que la de cigarrillos, en los últimos años diferentes estudios americanos nos revelan que en ese país las tasas de fumadores de puros están aumentando (Baker et al., 2000; Gilpin y Pierce, 1999; Shapiro, Jacobs y Thun, 2000), y más concretamente, entre personas jóvenes (Rigotti, Lee y Wechsler, 2000). Este hecho se achaca a un aumento de actividades promocionales a partir de 1992, encaminadas a incrementar el consumo de puros (Feit, 2001; Slade, 1998).

Hoy sabemos que la nicotina produce adicción (American Psychiatric Association, 2002; Organización Mundial de la Salud, 1992; USDHHS, 1988). La cantidad de nicotina de los cigarrillos oscila entre 0.07 y 1.4 mg. Los más vendidos en el mercado tienen un contenido entre 0.6 y $0.9 \mathrm{mg}$. Los puros contienen cantidades de nicotina que varían desde las cantidades contenidas en varios cigarrillos a las contenidas en uno o más paquetes de cigarrillos. La cantidad de nicotina es normalmente proporcional a la cantidad de tabaco contenida en el puro (Fant y Henningfield, 1998), oscilando entre 13 y 170 mg.; si su ingestión fuese total resultaría letal para el hombre. Afortunadamente, en la combustión se destruye la mayoría de la nicotina. En los cigarrillos, dos tercios de la nicotina van a la atmósfera y un tercio llega al fumador (Becoña, Palomares y García, 1994). En los puros no se produce una ingestión tan importante de nicotina como en los cigarrillos, debido a que ésta se encuentra en forma de sales básicas, a diferencia de los cigarrillos que está en forma de sales ácidas. Estas sales básicas se disuelven en la saliva y se absorben por la mucosa bucal y por los plexos venosos sublingüales. Ello posibilita una baja absorción de nicotina, en proporción con el contenido que poseen los puros, y una escasa afectación a los pulmones de la nicotina presente en el humo inhalado de los puros. Esto hace que el fumador de cigarrillos tenga que inhalar profundamente para el mantenimiento del nivel de nicotina en sangre; mientras que el fumador de puros la obtiene sin necesidad de inhalar, y por tanto no le llegan tantas sustancias nocivas a los pulmones (Fant y Henningfield, 1998). De ahí que se considere que fumar puros sea menos nocivo para la salud que fumar cigarrillos.

Sin embargo, también existen diferencias en los patrones de consumo de los fumadores de puros. De hecho, la Asociación Americana de Psiquiatría (American Psychiatric Association, 1996) considera que la principal diferencia entre los fumadores de pipa y puros está en si su consumo es primario o secundario. Así mismo, Shanks y Burns (1998) distinguen entre fumador primario, que sólo fuma puros y que nunca ha fumado cigarrillos o pipa (sin historia anterior de consumo de cigarrillos); fumador secundario, el que sólo fuma puros pero anteriormente fumó cigarrillos y/o pipa, combinándolos con el consumo de puros o exclusivamente y; fumador mixto, aquel que fuma puros a la vez que cigarrillos o pipa. La importancia de esta diferencia radica en que cuando se trata de consumidores secundarios se fuma con más intensidad (USDHHS, 1988), y esto puede conllevar una mayor dependencia de la nicotina. Por lo tanto, tanto el fumar tabaco de pipa como puros resulta menos nocivo que los cigarrillos sólo en el caso de no tragar el humo, pero casi todos los viejos fumadores de cigarrillos lo inhalan voluntaria o involuntariamente.

Por otra parte, los consumidores de puros suelen ser varones y tener una media de edad superior a la de los consumidores de cigarrillos. La tipología de consumo, pues habitualmente se trata de fumadores secundarios o mixtos, junto con una edad mayor, lo que se deriva generalmente en una historia de consumo de más años, hace que sea más probable que sufran enfermedades relacionadas con el consumo de tabaco en general (ej., enfermedades cardiovasculares, cáncer de pulmón, de laringe, bronquitis crónica, etc.) y de puros en particular (ej., cáncer oral).

Aunque es muy probable que los consumidores de puros sean personas dependientes de la nicotina, no existen datos empíricos acerca de la dependencia de la nicotina o su tratamiento en este grupo de fumadores (Becoña y Vázquez, 1998b). La American Psychiatric Association (1996) considera que si nos basamos en el hecho de que la sustancia que causa la adicción es la misma que en los consumidores de cigarrillos, es lógico pensar que los fumadores de puros, que tienen establecida una dependencia, se beneficiarán de los mismos tratamientos recomendados para los fumadores de cigarrillos. Basándonos en esta idea, nos propusimos el llevar a cabo una intervención conductual (Becoña, 1993), de demostrada eficacia tanto a nivel clínico (ej., Becoña y Vázquez, 1997) como comunitario (Becoña y Vázquez, 2001; Míguez, Vázquez y Becoña, 2002) en fumadores de cigarrillos, adaptada para fumadores de puros. Hoy las técnicas conductuales son las que obtienen las mejores tasas de abstinencia en población general y en grupos específicos (ej. Becoña et al., 2001; Lando, 1993; Lichtenstein y Glasgow, 1992; USDHHS, 2000). Dentro de las técnicas psicológicas, los programas multicomponentes son el recurso más en boga para dejar de fumar. Esta modalidad de tratamiento, también conocida como paquete de tratamiento o tratamiento multimodal, engloba varias técnicas de tratamiento a lo largo de tres fases (Becoña, 1994a; Lichtenstein y Glasgow, 
1992): fase de preparación, de abandono y de mantenimiento.

A continuación, describimos el tratamiento de un fumador de puros, mediante un programa psicológico conductual multicomponente.

\section{EVALUACIÓN}

E.R.L. se puso en contacto con nosotros, a través de una llamada telefónica, para someterse a tratamiento para dejar de fumar. Se trataba de un varón de 48 años de edad, casado y de profesión periodista. Los motivos que le impulsaron a tomar la decisión de acudir a tratamiento para dejar de fumar fueron: en primer lugar, por un problema grave de salud (infarto) que había padecido y porque habitualmente se encontraba muy cansado; en segundo lugar, por lo desagradable que le resultaba la adicción psicológica que el consumo de tabaco le creaba, manifestando que le molestaba mucho el sentirse dependiente de una sustancia y; por último, por su hija de 7 años que se lo pedía a diario. Además, aunque no era uno de los principales motivos que le llevaron a plantearse dejar de fumar, era consciente del gran coste económico que le suponía su adicción, pues estimaba que su gasto medio mensual en tabaco era del orden de unas 40.000 pesetas. Por tanto, dejar de fumar le ayudaría a mejorar su calidad de vida y el grado de satisfacción personal.

Al igual que en otras conductas adictivas, en el tabaquismo la evaluación es un proceso indispensable antes, durante, al final del tratamiento y en los seguimientos. Para la evaluación antes del tratamiento se utilizó el Cuestionario sobre el hábito de fumar (Becoña, 1994b). Este instrumento está formado por un total de 58 ítems. En él se recogen las distintas variables demográficas, dirección del fumador, variables relacionadas con el consumo, intentos de abandono o reducción del consumo, razones para dejar de fumar en intentos previos, procedimientos que ha seguido para dejar de fumar, creencias sobre la incidencia del tabaco sobre la salud, enfermedades, síntomas y molestias que padece o ha padecido por fumar, consumo de alcohol, café y otros medicamentos, deseo de dejar de fumar y expectativas ante el programa de tratamiento. Además, se incluye la Escala para la evaluación de los estadios de cambio (McConnaughy, DiClemente, Prochaska y Velicer, 1989; McConnaughy, Prochaska y Velicer, 1983), el Test de Fagerström de dependencia de la nicotina (Heatherton, Kozlowski, Frecker y Fagerström, 1991) y la versión reducida de la Escala de confianza en situaciones de fumar (Baer y Lichtenstein, 1988).

E.R.L. fumaba una media de 5 puros y 7 cigarrillos diarios, cigarrillos que había empezado a consumir seis meses atrás. Hasta hacía cuatro años, antes de empezar a consumir puros diariamente, había estado fumando una media de 60 cigarrillos diarios de la marca Ducados. Su primer cigarrillo lo había probado a los 20 años, consolidando el hábito un año más tarde, a los 21 años. Por tanto, llevaba 27 años fumando regularmente. En el último mes refiere haber fumado 150 puros y 40 puritos, aproximadamente. Inhalaba el humo al fumar, aprovechando los puros hasta el final, aunque no sabía el número aproximado de caladas que le daba a cada uno.

E.R.L. vivía con su esposa e hija, ambas no fumadoras. Su padre fue fumador y sus hermanos también lo eran en el momento de la evaluación.

En el último año había dejado de fumar cuatro días, pero no había reducido su consumo ni había hecho un intento real de dejarlo. En el pasado, había hecho varios intentos para dejar de fumar, llegando a estar en una ocasión 12 meses abstinente (hace cuatro años). Uno de los motivos que le indujo a abandonar el tabaco fue el relacionado con la salud, siendo principalmente su médico el que le incitó a ello, el cual le había sugerido en repetidas ocasiones que debía dejar de fumar. E.R.L. también pensaba que el fumar afectaba muchísimo a la salud y le molestaba, especialmente, la dependencia que le creaba el tabaco.

Respecto a enfermedades y síntomas, indicó que había padecido "fatiga, falta de aire, continuos resfriados y secreciones mucosas"; como enfermedades importantes había sufrido un infarto agudo de miocardio. Ultimamente, notaba fatiga, cansancio, ansiedad y sensación de dependencia, como molestias más importantes que le producía el tabaco. Además, se trataba de una persona que practicaba deporte habitualmente y en esos momentos era cuando más acusaba la fatiga y la falta de resistencia. Se encontraba a tratamiento médico para la hipertensión y el colesterol y a excepción de los problemas que achacaba al tabaco, no padecía ni había padecido ninguna otra enfermedad de importancia.

Con respecto al consumo de alcohol, E.R.L. indicó que por semana no solía tomar bebidas alcohólicas, sin embargo, el fin de semana consumía una media de 4 cañas, 4 vasos de vino, 4 copas de champán o sidra y 2 copas de bebidas de alta graduación. Además, consumía un promedio de 3 cafés diarios.

Sus deseos de dejar de fumar y de realizar el tratamiento eran altos, puntuando 8 en una escala de 0 (nada) a 10 (máximo), y tenía buenas expectativas sobre el programa de tratamiento para dejar de fumar que iba a realizar, aunque no conocía a ninguna persona que hubiese venido con anterioridad a tratamiento. En el último año había dejado de fumar en una ocasión al menos 24 horas y, en el momento de la evaluación, tenía la intención de dejarlo dentro de los siguientes 30 días. Esto es un índice de que el sujeto 
se encontraba en el estadio de preparado para la acción (Prochaska, Norcross y DiClemente, 1994). En éste, el fumador considera la conducta de fumar como un problema y piensa seriamente en la posibilidad de cambiar a corto plazo. En el test de Fagerströn de dependencia de la nicotina (Heatherton, Kozlowski, Frecker y Fagerström, 1991) obtuvo una puntuación de 7, lo cual indica una elevada dependencia fisiológica.

Un dato interesante es la evaluación de la eficacia que percibe el sujeto de mantenerse abstinente en aquellas situaciones en las que tiene mas probabilidad de fumar; esto es, su autoeficacia (ej., Condiotte y Lichtenstein, 1981). De la Escala de confianza en situaciones de fumar de Condiotte y Lichtenstein (1981) se elaboró una versión reducida (Baer y Lichtenstein, 1988) de 14 ítems, el Cuestionario de resistencia de la urgencia a fumar. En ella se indican 14 situaciones en las que la gente fuma con más frecuencia. El sujeto tiene que indicar la probabilidad de su resistencia a la urgencia a fumar de 0 a 10 , en donde 0 es lo equivalente a ninguna resistencia y 10 a total resistencia, con un punto intermedio de 5. En dicha escala, E.R.L. obtuvo una puntuación total de 43.

E.R.L. también cumplimentó varias escalas relacionadas con una evaluación complementaria (ansiedad, estrés y depresión). Dichas escalas fueron las siguientes: el Inventario de la depresión de Beck (Beck, Rush, Shaw y Emery, 1979), la Escala de estrés percibido (Cohen, Kamarck y Merlmenstein, 1983) y la Escala de Ansiedad-Rasgo de Spielberger (Spielberger, Gorsuch y Lushene, 1970). Destacar que en el Inventario de la depresión de Beck, obtuvo una puntuación total de 15 puntos y en la escala de Ansiedad-Rasgo ocupaba el decatipo 6.

También se le evaluó el monóxido de carbono en aire espirado (CO), observándose una cifra de 55 partículas por millón (ppm) (Beldfont Instruments, Sittingbourne, Kent, UK). Este proceso de medición y corroboración con esta medida fisiológica se repitió en las sesiones de tratamiento y en las de seguimiento.

Se le explicó brevemente en que consistía el tratamiento para dejar de fumar, aclarándole que el objetivo era que dejara de fumar tratando las dependencias fisiológica, psicológica y social con un programa de corte psicológico, dado que el consumo de tabaco es una adicción que está mantenida por estos tres factores.

Al final de la sesión de evaluación se le proporcionaron los autorregistros que se utilizan en nuestro tratamiento (Becoña, 1993). Los autorregistros son la medida conductual más utilizada en el tratamiento de los fumadores. En las hojas de autorregistro el fumador tiene que ir anotando cada cigarrillo, puro y/o purito y distintos aspectos de la conducta de fumar (ej., hora de consumo, placer que le proporciona de 0 a 10 , situaciones en las que fuma). Este sujeto realizó un total de 10 días de autoobservación para la línea base y, posteriormente, siguió registrando su consumo diario hasta que dejó de fumar. En este caso, puesto que se trata de un consumidor diario de puros, fue necesario realizar una pequeña modificación, con el objetivo de adaptar el registro al consumo de puros. Los puros no suelen consumirse de una sola vez, sino que se apagan y se encienden en varias ocasiones, por lo tanto E.R.L. debía registrar de cada puro las veces que lo encendía, anotando la hora, placer y situación como si se tratara de cigarrillos o puros distintos.

\section{TRATAMIENTO}

Se le aplicó el Programa para Dejar de Fumar (Becoña, 1993) a lo largo de 6 sesiones de tratamiento, adaptado para este caso de fumador de puros. Este tratamiento consta de los siguientes componentes: contrato de tratamiento, autorregistros, representación gráfica del consumo, información sobre el tabaco en general y el consumo de puros en particular, reducción gradual de ingestión de nicotina y alquitrán, control de estímulos, estrategias para no padecer el síndrome de abstinencia de la nicotina, feedback fisiológico del monóxido de carbono en el aire espirado y entrenamiento en estrategias de prevención de la recaída. Las cuatro primeras sesiones se centraron en eliminar las dependencias fisiológica, psicológica y social del sujeto al tabaco. Las sesiones quinta y sexta fueron dedicadas a tratar aspectos relevantes relacionados con la prevención de la recaída y el mantenimiento de la abstinencia.

\section{Primera sesión}

Al comienzo de la sesión se le entregó el material de la primera sesión de tratamiento y la hoja para la representación gráfica del consumo. A continuación, se le explicó las características del tratamiento, se tomaron las medidas de monóxido de carbono (ver Figura 1) y se analizaron los registros que había realizado a lo largo de los días de la línea base (desde la sesión de evaluación), anotándose el consumo diario de puros y cigarrillos desde ese momento. E.R.L. informó de que no había realizado todos los días los registros, pues a veces se le olvidaban en casa. Hay que tener en cuenta que los autorregistros poseen un formato adaptable al paquete de cigarrillos, y es precisamente aquí donde los fumadores deben llevarlos, para evitar el olvido de su uso. Sin embargo, en este caso al tratarse de un fumador de puros no existe esta posibilidad (los cigarrillos que consumía los pedía, nunca los compraba). A partir de aquí, se le recomendó a E.R.L. que llevara los registros en la billetera.

Se enseñó al sujeto a analizar los antecedentes y consecuentes del consumo de tabaco, explicándole que los antecedentes de su conducta de fumar se 
evalúan analizando sus autorregistros y viendo en qué situaciones fumaba, con qué personas, en qué momentos del día, etc. Se le explicó que los consecuentes de su conducta de fumar eran los efectos que le producía el acto de fumar o el tabaco por sí mismo (la nicotina que contiene). Analizar los antecedentes y consecuentes de la conducta de fumar resulta necesario de cara a buscar las situaciones de alto riesgo del sujeto, mediante el placer que experimenta en las mismas cuando fuma, o a través de la frecuencia de consumo en ellas. Una vez identificadas, se entrena al sujeto en distintas estrategias para hacerles frente, utilizando la técnica de control de estímulos.

Se trataron brevemente las consecuencias del tabaco sobre la salud, proporcionándole material escrito al respecto (apéndices A y B de Becoña, 1993) y se discutieron las razones para fumar y para no fumar, así como los beneficios de dejar de fumar.

En esta primera sesión, también se le explicó cómo hacer la representación gráfica de su consumo. Se le entregó al comienzo de la sesión una gráfica explicándole que en el eje de abscisas se representaba el número de puros fumados y, en el de ordenadas, los distintos días del mes. Al tratarse de un consumidor de puros, la diferencia a nivel cuantitativo en el consumo diario apenas es perceptible, si utilizamos la gráfica del consumo de cigarrillos. Por lo tanto, basándonos en esta gráfica, se le indicó a E.R.L. que tomara como referencia el 10 (cigarrillos) como equivalente a 1 puro, el 20 a 2 puros y así sucesivamente. De esta forma la gráfica cumpliría su función, pues permitiría ver las oscilaciones que se dieran en el consumo a lo largo del tratamiento.

Se le hizo ver la necesidad de tomar un papel activo y público en el abandono del tabaco. Para ello, era necesario que le comunicase a las personas de su entorno, con las que vivía o se relaciona habitualmente (familia- res, amigos, compañeros de trabajo), que iba a dejar de fumar en los próximos 30 días. Esto facilitaría la implicación activa del sujeto en el cambio de su conducta y el poder analizar tanto las reacciones de esas personas como las suyas ante la propuesta de dejar de fumar.

A continuación se le explicó que, cuando se trata de un fumador de cigarrillos, se utiliza el cambio de marcas para poder reducir el contenido de nicotina y alquitrán de sus cigarrillos en un 30\% para la siguiente semana, sin embargo, en su caso, teníamos que conseguir esa reducción siguiendo otra estrategia, pues la marca de los puros no nos resulta indicativa de la nicotina que contienen. La cantidad de nicotina es normalmente proporcional a la cantidad de tabaco contenida en los puros (Fant y Henningfield, 1998) y existe una enorme variabilidad entre estos en cuanto a tamaño, grosor y peso. Por ello, resultaba importante que E.R.L. consumiera siempre los mismos puros, para poder lograr el control y la reducción paulatina de la ingestión de nicotina. Al analizar los registros realizados durante la línea base, se observó que el consumo de E.R.L., a lo largo de este tiempo, había estado oscilando entre 4-5 puros y 5-7 cigarrillos, cigarrillos que fumaba exclusivamente por las mañanas, pues los pedía a sus compañeros de trabajo. Así pues, de cara a la siguiente semana, se le recomendó eliminar el consumo de esos cigarrillos y fijar el consumo de puros en un máximo de 4 al día.

También se le dieron varias reglas prácticas para que la reducción del consumo fuese efectiva y para inmunizarlo a la presión social: no aceptar ofrecimientos de tabaco, dejar el último tercio del puro sin fumar, reducir la profundidad de las inhalaciones y llevar el puro a la boca sólo para fumarlo.

Por último, se recapituló todo lo expuesto, se le entregaron autorregistros para la semana y se le explicó como utilizarlos para adaptarlos al consumo de puros.

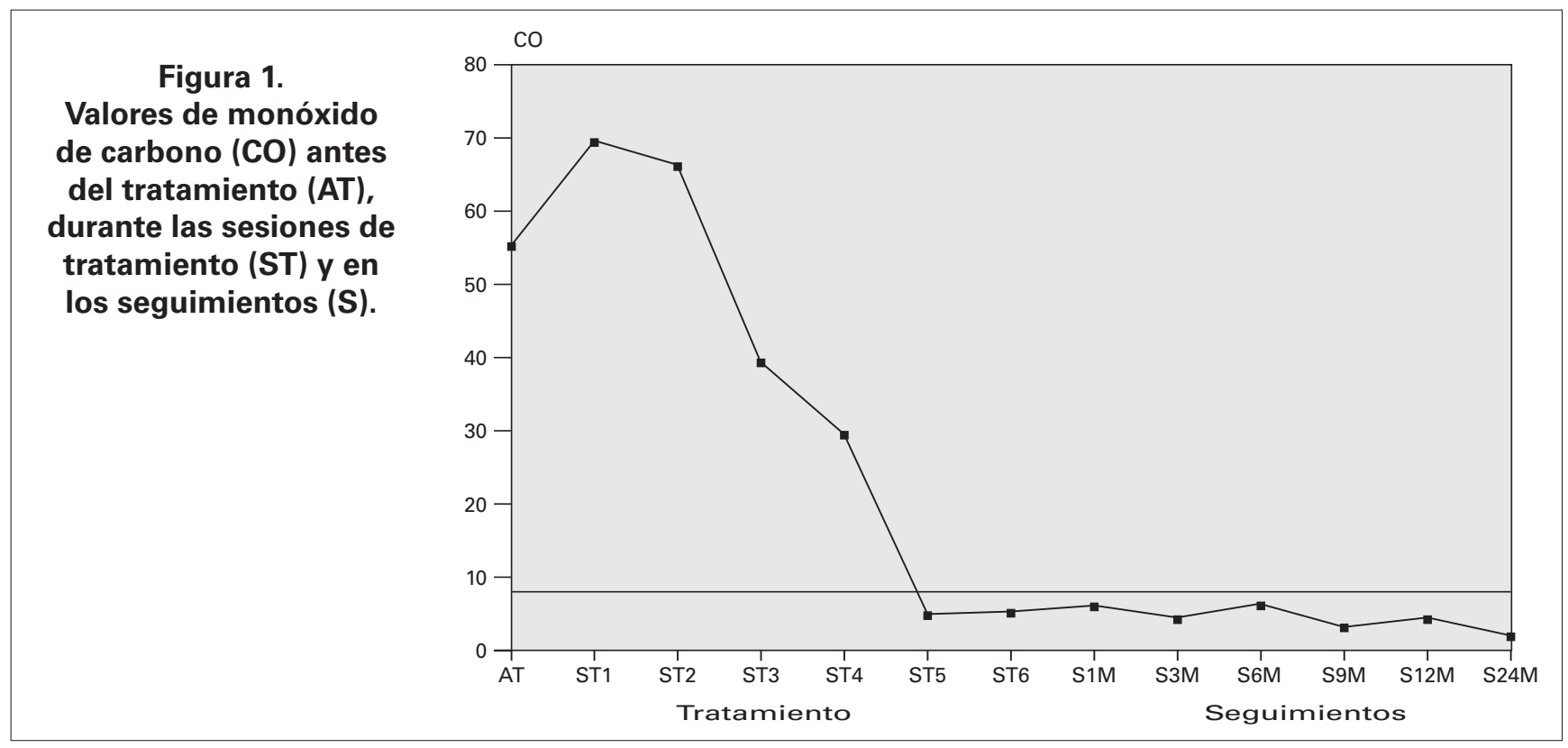




\section{Segunda sesión}

Al comienzo de la sesión se tomó la medida de monóxido de carbono, se anotó el consumo de puros desde la sesión anterior y se le proporcionaron nuevos autorregistros.

Se comenzó la sesión con un análisis de los objetivos propuestos en la primera sesión y la contestación a las posibles dudas surgidas (reglas prácticas, consecuencias del tabaco para su salud, etc.). E.R.L. no se encontraba demasiado satisfecho, porque había logrado realizar las metas previstas durante la semana pero, llegado el domingo, fumó sin control. Así mismo, se observó que el sujeto presentaba una concentración de monóxido de carbono muy alta y similar a la sesión anterior. En la primera sesión la cantidad de $\mathrm{CO}$ fue de 69 ppm; en ésta de 65 p.p.m (ver Figura 1). Ante este hecho, se le explicó que el dejar de fumar es un proceso en el cual puede haber altos y bajos y lo importante es no caer en el desánimo. Se analizó la situación que le llevó a fumar sin control el domingo y se le animó a que utilizara este incidente como una experiencia de aprendizaje, de cara a prevenir problemas similares en el futuro.

También se comentó cómo había aplicado las reglas prácticas proporcionadas para reducir el consumo de tabaco, indicándole que siguiera utilizando las mismas y que introdujera algunas nuevas: realizar registros; aumentar la parte no fumada de cada puro; no aceptar ofrecimientos de cigarrillos y/o puros; $y$, retrasar el primer consumo del día hasta 15 minutos después de levantarse o desayunar. E.R.L. había aplicado correctamente todas las estrategias sugeridas en la sesión anterior a excepción de dejar un tercio sin fumar, pues consideraba que no era capaz de ello. Se le explicó la importancia de cumplir esta tarea de cara a la próxima semana, recomendándole que marcara los puros previamente a su consumo pues esto le ayudaría a recordar en que momento debía parar.

En esta sesión el terapeuta analizó los autorregistros conjuntamente con el sujeto, prestando especial atención a los antecedentes y consecuentes. Los antecedentes que elicitaban la conducta de fumar eran conducir el coche, leer, hablar por teléfono (sólo cuando efectuaba una llamada), escribir en el ordenador, cuando se encontraba en tertulia, con el café, después de la comida, cuando estaba descansando y cuando intentaba concentrarse. Como consecuentes positivos aparecían el aumento de la sensación de bienestar, de relax, sentimiento de que era más fácil hacerle frente a una determinada situación, mayor concentración, sensación de ensoñación y evasión, y reducción o eliminación del síndrome de abstinencia. E.R.L. destacó como consecuentes negativos los riesgos que suponía fumar para su salud y el gran cansancio a que le conducía.
La conducta de fumar, por tanto, estaba bajo el control de numerosos estímulos antecedentes. Teniendo en cuenta que la probabilidad de una respuesta se puede aumentar o disminuir mediante la presentación o retirada de dichos estímulos, se introdujo la técnica de control de estímulos, cuya finalidad en nuestro caso era disminuir la conducta no deseada mediante la restricción del control estimular. Como hemos visto, E.R.L. fumaba en situaciones concretas, cuando realizaba determinadas actividades, cuando experimentaba determinados estados emocionales, etc. A partir de esta sesión comenzamos a restringir el consumo de puros en alguna de esas situaciones. En concreto, en aquellas en las que le resultaba más fácil dejar de fumar, para ir en las siguientes sesiones eligiendo aquellas situaciones que ahora le eran subjetivamente más difíciles, pero que a medida que se va avanzando en el proceso van siendo consideradas más fáciles por el sujeto.

Ya centrándonos en la aplicación práctica del procedimiento, eligió las tres situaciones (paseando, esperando y escribiendo a ordenador) en las que dejaría de fumar a partir del día siguiente, y que consideraba las más fáciles de cumplir, de todas aquellas que tenía registradas en sus autorregistros.

Respecto a la reducción de puros se le indicó el número de puros máximos que podía fumar (3) y se le sugerió que prescindiera de fumar en aquellos momentos que valoraba con un placer inferior a 5 (la escala del autorregistro va de 0 a 10).

Aunque ya se observaron avances en el proceso de dejar de fumar, a partir de esta sesión suelen ser mucho mayores. Se le sugirieron una serie de actividades (beber agua abundantemente, tomar zumos, reducir el consumo de alcohol y café, etc.) para evitar el síndrome de abstinencia, indicándole que escogiera varias y las pusiera en práctica durante la siguiente semana.

Respecto a la dependencia social, se hizo un breve comentario de las reacciones que percibió el sujeto, acerca del apoyo o no, a su abandono del tabaco. El apoyo facilita o favorece el abandono y el superar momentos difíciles que se puedan presentar. Se le recomendó que prosiguiera para la siguiente semana analizando esas reacciones y las suyas propias a los comentarios de otra gente y de la publicidad en torno al tabaco.

Finalmente, se hizo una recapitulación de todo lo expuesto.

\section{Tercera sesión}

Al comienzo de la sesión se tomó la medida de monóxido de carbono, se anotó el consumo realizado desde la segunda sesión y se le entregaron autorregistros nuevos, para que los cumplimentara en la 
semana siguiente. E.R.L. comentó cómo le había ido a lo largo de la semana e informó que la evaluación de CO le era de gran ayuda y que le animaba a seguir disminuyendo su consumo (al observar que en esta sesión se había reducido casi a la mitad con respecto a la sesión anterior, pasando de 65 a 37 p.p.m.). Asimismo, destacó que había experimentado una notable mejoría en el plano físico, no se fatigaba tanto cuando hacía ejercicio, pero tenía más tos y un incremento de secreciones y sensaciones molestas en la garganta. El terapeuta hizo un breve comentario acerca de su mejoría física y de algunas sensaciones que estaba experimentando que eran sinónimas de regeneración corporal (incremento de tos, carraspera....). Éstas eran claros indicios de que su cuerpo empezaba a recuperarse. Antes, al fumar, tenía atrofiadas algunas funciones de sus órganos vitales y ahora había comenzado el proceso de limpieza de sus pulmones. Se hizo explícito de que de ningún modo se podía equiparar este tipo de sensaciones con un empeoramiento físico, sino todo lo contrario.

Se siguió la sesión analizando cómo había aplicado las reglas prácticas que se le habían indicado para reducir las dependencias fisiológica, psicológica y social producidas por el tabaco. E.R.L. había seguido sistemáticamente todas las tareas sin encontrar mayor dificultad en el cumplimiento de las mismas. Se le recomendó seguir haciendo los autorregistros, la representación gráfica y no aceptar ofrecimientos de cigarrillos y/o puros.

Para esta semana tenía que limitar su consumo a 2 puros diarios e incrementar la parte del puro que dejaría sin fumar, es decir, la mitad. La razón de esta regla se debe a que el disminuir la ingestión de nicotina puede llevar al fumador a efectuar el proceso denominado "compensación", que consiste en tratar de compensar la nicotina que se deja de ingerir, en este caso a través de la disminución del número de puros, dando un mayor numero de inhalaciones o haciendo que éstas sean más profundas. Con esta regla se evita el proceso de compensación nicotínica. Además, debía retrasar un mínimo de 30 minutos el primer puro de la mañana, de después de levantarse o de desayunar. Tarea que no le representaba ninguna dificultad pues solía hacer su primer consumo cuando llegaba al trabajo, una hora después de haberse levantado. Por lo que respecta al consumo de después de comer y de cenar tenía que retrasarlo 15 minutos. Esta tarea tiene por objetivo el ir distanciando dos conducta que se encuentran muy asociadas, como es el acabar de comer y fumar. Con ello lograremos que poco a poco se vaya perdiendo esa asociación.

En cuanto a la reducción de la dependencia psicológica, E.R.L. había dejado de fumar en las situaciones acordadas en la semana anterior sin ninguna dificultad (control de estímulos). Así mismo, escogió otras tres situaciones para no fumar la siguiente semana: estu- diando/leyendo, trabajando, hablando por teléfono. Se le animó a que aplicara las mismas estrategias que le habían ayudado a eliminar algún consumo en la semana anterior, o alguna otra de las que se le habían sugerido (hacer inspiraciones profundas y expulsar el aire lentamente, dar paseos, beber agua, etc.).

En lo concerniente a la dependencia social, E.R.L. hizo un breve comentario de las reacciones que percibió acerca del apoyo o no al abandono del tabaco en su entorno. Comentó que en la semana anterior recibió un buen apoyo, tanto en casa como en el trabajo, aunque los compañeros de trabajo se manifestaban un poco escépticos

\section{Cuarta sesión}

Tras 7 días desde la última sesión, E.R.L. acudió de nuevo a tratamiento. Se le evaluó el monóxido de carbono, que fue de 25 p.p.m. (ver Figura 1), se registró el consumo de puros a lo largo de la semana y se le proporcionaron más hojas de autorregistro.

Se inició la sesión con un comentario general acerca de la existencia de algún problema que se le hubiese planteado al sujeto a lo largo de la semana. Destaca su preocupación por la posibilidad de engordar, pues se queja de que desde que ha disminuido el consumo de tabaco ha incrementado el consumo de bombones, gominolas y todo tipo de productos dulces. Se abordó con él esta situación, reconsiderando que si bien era positivo que se mantuviese alerta acerca del incremento en el consumo de ciertos productos que podían conllevar un aumento de peso, su preocupación por el momento no tenía fundamento, por razones objetivas: 1) hasta el momento no había ganado peso y, 2) era probable que esta apetencia por el dulce fuese algo puntual y se estabilizara en pocos días.

E.R.L. se encontraba muy motivado para dejar de fumar y creía que era posible conseguirlo, pues ya lo había logrado con anterioridad. Sin embargo, tenía dudas de si conseguiría luego mantenerse abstinente. Se le explicó que el logro de la abstinencia y del mantenimiento eran dos procesos distintos y que el segundo implicaba necesariamente la consecución del primero. Por lo tanto, en primer lugar nos íbamos a centrar en conseguir dejar de fumar y una vez logrado esto, nos centraríamos en las estrategias a seguir de cara al mantenimiento de la abstinencia.

E.R.L. hizo un comentario acerca de las múltiples ventajas que ya le había acarreado la reducción del consumo de tabaco: se sentía mejor físicamente (respiraba mejor, no se fatigaba cuando practicaba deporte...), también apreció una mejoría en los sabores y en los olores, lo que se derivaba en una mayor apetencia por la comida. Se le recomendó que pensara en cómo creía que se iba a sentir como no fumador, y que 
repasara las ventajas que había logrado hasta ese momento.

Posteriormente, se comentó la aplicación de todas las reglas prácticas que había utilizado para reducir el consumo de puros. Se le recomendó que siguiera haciendo los registros que, de cara a la próxima semana, fumara como máximo la mitad de un puro, no aceptara ofrecimientos de ningún producto del tabaco, retrasara 45 minutos el primer consumo de después de levantarse y del desayuno y que no fumara hasta un mínimo de 30 minutos después del café de la mañana, de después de comer y de cenar. El objetivo era que a lo largo de la semana fuese reduciendo su consumo paulatinamente, hasta conseguir dejar de fumar al final de la misma. Para ello, además de lo comentado, debería eliminar las dos situaciones que le quedaban (después de comer y viendo la TV), dentro de dos días una y dentro de cuatro o cinco la última. Se le animó a que siguiera aplicando algunas de aquellas estrategias que le habían sido útiles para reducir más fácilmente el consumo (caminar, pasear, hacer inspiraciones profundas, etc). También se le dijo al sujeto que debería pensar en darse algún "premio" cuando lograra la abstinencia.

Respecto a la dependencia social, se le recomendó que no la obviase, que la siguiese controlando, siendo consciente de las presiones de tipo social que recibe, sobre todo en las comidas de trabajo que para E.R.L. representaban la situación de mayor riesgo y preocupación.

Se hizo un análisis de la evolución del impulso a fumar y de cómo vencerlo. Se observó que a lo largo del tratamiento, como era de esperar, el impulso o necesidad a fumar de E.R.L. había disminuido en frecuencia e intensidad. En la mayoría de las situaciones ya no experimentaba esa necesidad de fumar. Ahora estos impulsos o tentaciones a fumar se limitaban sólo a algunas situaciones y duraban escasos segundos. Se le recomendó que en dichas situaciones tratara de pensar que sólo se trataba de "aguantar" unos pocos segundos y que transcurridos éstos la tentación o impulso a fumar remitiría totalmente. Si esto no le resultase suficiente, se le indicó que practicara el realizar inspiraciones profundas y, a continuación, expulsara el aire lentamente.

Así mismo, se recalcó el hecho de que la finalidad del tratamiento era que él consiguiera el autocontrol de su conducta de fumar. Se le señaló que el objetivo final que pretendíamos era que dejara de fumar y que luego se mantuviese abstinente, controlando su propia conducta. Para conseguir esto, él estaba aprendiendo una serie de estrategias que le estaban ayudando a conseguir un control progresivo de su conducta de fumar, como ya había podido observar. Se le señaló que antes de realizar el tratamiento él fumaba sin ningún tipo de control, estando a merced de la necesidad de nicotina que tuviese el organismo. Según fue trans- curriendo el tratamiento no sólo rebajó el consumo y la dependencia a la nicotina, sino que también, y lo que era más importante, ahora ya casi era capaz de controlar totalmente su conducta de fumar.

En esta sesión también se introdujo lo que era una creencia errónea y se ejemplificó con la más importante que suelen tener los fumadores: la equiparación de caída con recaída. Se le pidió que reflexionara sobre la distinción entre caída y recaída y sus creencias acerca de esta cuestión.

Finalmente, se le resumió las tareas que tenía que realizar para la siguiente semana y se quedó con él para la próxima sesión de tratamiento.

\section{Quinta y sexta sesión}

Como en las sesiones anteriores, al comienzo de cada sesión se le evaluó el monóxido de carbono. Como puede observarse en la Figura 1, éste se redujo considerablemente con respecto a las sesiones previas, siendo en ambas sesiones de 5 p.p.m

En la quinta sesión E.R.L. mostró una gran satisfacción porque llevaba 24 horas sin fumar. El terapeuta aprovechó este hecho para hacer énfasis en los beneficios que ya estaba notando al haber dejado de fumar (mejoría física y psicológica, junto a una gran satisfacción interna por haberse liberado de una dependencia). También se le comentó que no se debía preocupar si ahora le surgían dudas sobre lo que le costaría mantenerse abstinente en el futuro. Estas dudas van desapareciendo conforme transcurren los días como no fumador. Por ello, lo importante en este momento es que se plantee la abstinencia día a día.También se discutió la importancia de irse adaptando poco a poco a la situación de ser un no fumador y de que ahora tenía que hacerse consciente de que había dejado de fumar. Pasar de fumar todos los días a no fumar es un proceso que exige un período de adaptación.

Tanto la quinta como la sexta sesión se orientaron al mantenimiento de las ganancias terapéuticas. En ambas sesiones se comentaron algunas sensaciones que podía percibir estos primeros días sin tabaco: tener la sensación de que le faltaba algo, ganas de fumar, sequedad de boca, sabor a nicotina, entre otras. Así mismo, se analizaron las creencias erróneas más importantes que suelen llevar a los sujetos a la recaída: al dejar de fumar, ¿se vuelve uno más ansioso o nervioso, irascible o agresivo?, ¿se gana peso?, ¿se pierde concentración?, ¿si se fuma un cigarrillo ya se ha vuelto a recaer?.

Al final del tratamiento, E.R.L. completó el Cuestionario de evaluación de final de tratamiento (Becoña, 1994b) que incluye ítems acerca del estatus de fumador, apoyo social, mejoría física y psicológica y la Escala de síntomas de la abstinencia del tabaco de Hughes 
y Hatsukami (1986). En la evaluación del final del tratamiento se estimó que el sujeto estaba abstinente, pues no había fumado desde las 24 horas previas a la quinta sesión de tratamiento y el nivel de monóxido de carbono en el aire espirado fue menor a 9 ppm (ver Figura 1), tanto en la quinta como en la sexta sesión.

A continuación, se le felicitó por la consecución de la abstinencia y se le animó de cara al futuro, recordándole que este tratamiento se dirigía al autocontrol de su conducta, habiendo aprendido todo un conjunto de estrategias que le permitirían seguir sin fumar. Finalmente, se acordó la fecha del primer seguimiento para un mes más tarde.

\section{Seguimientos}

Se hicieron seguimientos a 1, 3, 6, 9, 12 y 24 meses después de finalizado el tratamiento. Al mes y a los 3 meses, se evaluó la abstinencia con autoinformes validados con medidas de monóxido de carbono. También se analizaron los problemas que le fueron surgiendo al sujeto. A los 6, 9, 12 y 24 meses se utilizó el Cuestionario de seguimiento (ver Becoña y Míguez, 1995).

Para cada tiempo de seguimiento hemos utilizado un criterio específico de abstinencia, siguiendo las recomendaciones para la abstinencia de Velicer, Prochaska, Rossi y Snow (1992). Al final del tratamiento, 1 y 3 meses de seguimiento el sujeto tenía que llevar un mínimo de 24 horas abstinente. A los 6 y 9 meses se requería que el sujeto llevase al menos 7 días sin fumar ningún producto del tabaco (cigarrillos, pipas, puros, etc.). A los 12 y 24 meses de seguimiento que no hubiera fumado nada en los 30 días anteriores. E.R.L cumplió todos los requisitos anteriores para la abstinencia puntual. Además, en el último seguimiento realizado, a los 24 meses, cumplía el criterio de abstinencia continua, es decir, no haber probado ningún producto del tabaco desde el final del tratamiento hasta dos años después. La información obtenida a través de los autoinformes, en los respectivos seguimientos, se cotejó con las medidas bioquímicas obtenidas por el monóxido de carbono en el aire espirado, que fueron inferiores siempre a 9 ppm (ver Figura1).

En los distintos seguimientos realizados, E.R.L. indicó que se sentía muy satisfecho de sí mismo por haber dejado de fumar; además de encontrarse muy bien tanto a nivel físico (tenía menos cansancio y respiraba mejor) como psicológico. En el seguimiento de los 24 meses, indicó haber ganado en torno a 9 kg, lo cual era algo que aunque inicialmente le preocupaba ahora no le daba demasiada importancia, pues aún con esos kilos de más se encontraba mejor que cuando fumaba. Además, el incremento de peso no lo achacaba sólo a haber dejado de fumar, pues tenía una mayor apetencia por la comida, sino también a que había dejado de practicar deporte con asiduidad.

\section{DISCUSIÓN}

El consumo de tabaco en cualquiera de sus presentaciones constituye un riesgo elevado para la salud y todos los fumadores pueden obtener importantes beneficios, si dejan de fumar (USDHHS, 1990). Diferentes estudios concluyen que el fumar cigarros puros es también una causa de cáncer (ej., Baker et al., 2000) y representa un importante riesgo para la salud. Sin embargo, a pesar de esto, no existe una política reguladora en torno a su consumo, como ocurre con los cigarrillos. Así, por ejemplo, a diferencia de los cigarrillos, los puros no llevan una etiqueta de advertencia de lo peligroso de su consumo (Connolly, 1998), auque sí los puritos, ni de su contenido en nicotina. Además, en relación al consumo de puros, tradicionalmente han existido una serie de creencias que lo vinculaban a una alternativa segura al consumo de cigarrillos, creencias que han propiciado el hecho de que los fumadores de puros tuviesen una menor percepción de riesgo personal de contraer cáncer (Baker, 2001) o la percepción de un menor riesgo de desarrollar adicción a la nicotina (Baker et al., 2000).

El humo de los puros contiene los mismos componentes tóxicos y carcinogénicos que se han identificado en el humo de los cigarrillos. La nicotina del humo del tabaco se absorbe con facilidad en los pulmones y no importa si este humo procede de cigarrillos o de puros. El nivel de exposición al humo de los puros se suele medir en función del número de puros que se consuman por día, lo cual se considera una medida poco precisa, debido a la variedad de tamaños de los puros (Sanks y Burns, 1998).

Hasta el momento actual, existen pocos estudios dirigidos a conocer específicamente la problemática del consumo de puros y los pocos existentes se centran en el estudio a nivel epidemiológico de la prevalencia de su consumo (ej., Gerlach et al., 1998) o los riesgos de salud asociados al mismo (ej., Baker et al., 2000; Shanks y Burns, 1998) pero, en ningún caso, en la intervención. De ahí que el objetivo de este trabajo ha sido proporcionar información de la problemática del tabaquismo en un fumador de puros y el abordaje del mismo, para su abandono, a través de la aplicación de un programa psicológico conductual multicomponente (Becoña, 1993) que, como hemos visto, se adaptó perfectamente a las necesidades del sujeto y le ayudó a dejar de fumar con éxito. Además, en este trabajo se pretende ilustrar una nueva aplicación de un tratamiento ya conocido y previamente evaluado en diferentes formatos (clínico, de autoayuda) y poblaciones (mujeres, personas mayores). Nuestros resultados indican que se trata de un tratamiento que se adapta perfectamente al consumidor de puros. Para ello, sólo es necesario realizar sencillas adaptaciones (del registro y de la gráfica) y la no consideración de marcas de cara a lograr la reducción de la dependencia fisiológica. Esto nos muestra, una vez más, la gran versatilidad de este tratamiento. 
Como hemos podido observar, en este caso, el proceso de romper con el tabaquismo conlleva una serie de pasos. En primer lugar, el fumador tiene que percatarse de su problema y decidirse a abandonarlo. En segundo lugar, tiene que dejar de consumir el tabaco. Y, en tercer lugar, romper con el hábito. En este aspecto, las estrategias cognitivo-conductuales son herramientas básicas para realizar aquellos cambios en la vida del fumador que le permitan permanecer sin fumar.

Respecto al tratamiento del tabaquismo, en las dos últimas décadas, se han producido progresos muy importantes. Actualmente hay una gran variedad de tratamientos (USDHHS, 2000). El problema es que del amplio abanico de posibilidades que existen hasta el momento no ha sido explorado en este grupo de población, como lo demuestra la inexistencia de estudios al respecto. Además, a un fumador de puros puede resultarle más difícil, si cabe, dejar de fumar porque el hábito está muy cronificado, pues suele tratarse de fumadores de mayor edad y, en la mayoría de los casos, tienen establecida una tolerancia importante a la nicotina, pues lo más habitual es que se trate de un fumador secundario o mixto, como el caso que nos ocupa. Recordar que E.R.L. tenía una alta dependencia a la nicotina, lo que se desprende de su patrón de consumo (aprovechamiento del puro hasta el final, inhalaciones muy profundas), de la puntuación obtenida en el Test de Fagerström de dependencia de la nicotina y del elevado nivel de CO que se observó tanto en la sesión de evaluación como en las primeras sesiones de tratamiento. El nivel de CO guarda relación tanto con el patrón de consumo como con la cantidad de tabaco que se fuma. Así, a modo de ejemplo, en un estudio realizado al respecto (Becoña y Vázquez, 1998a) se obtuvo, para una muestra de varones fumadores duros (consumo medio de 27 cigarrillos), una media de $\mathrm{CO}$ en la sesión de evaluación de 32 p.p.m.

Finalmente, hay que indicar que en la actualidad no disponemos de tratamientos para dejar de fumar evaluados en la población de consumidores de puros. La realidad es que necesitamos más información para hacer frente a este problema y ayudar a todo tipo de fumadores a que mejoren su calidad de vida dejando de fumar. De este modo, podremos determinar qué estrategias terapéuticas son las más adecuadas para el problema en cuestión y qué componentes funcionan mejor en nuestras intervenciones con este tipo de fumadores.

\section{REFERENCIAS BIBLIOGRÁFICAS}

American Psychiatric Association (1996). Practice guideline for the treatment of patiens with nicotine dependence. American Journal of Psychiatry, 153 (Suppl.), s1-s31.
American Psychiatric Association (2002). Manual Diagnóstico y Estadístico de los trastornos mentales $\left(4^{\circ}\right.$ edicición-Texto revisado) (DSM-IV-TR). Barcelona: Masson.

Baer, J.S. y Lichtenstein, E. (1988). Cognitive assessment in smoking cessation. En D.M. Donovan y G.A. Marlatt (Eds.), Assessment of Addictive Behaviors (pp.189-213) Nueva York: Guildford.

Baker, F., Ainsworth, S.R,. Dye, J.T., Crammer, C., Thun, M.J., Hoffmann, D., Repace, J.L., Henningfield, J.E., Slade, J. Pinney, J., Shanks, T., Burns, D.M., Connolly, G.N. y Shopland, D.R. (2000). Health risks associated with cigar smoking. JAMA, 284, 735-740.

Baker, F., Dye, J.T., Denniston, M.M. y Ainsworth, S.R. (2001). Risk perception and cigar smoking behavior. American Journal of Health Behavior, 25, 106-114.

Beck, A.T., Rush, A.J., Shaw, B.F. y Emery, G. (1979). Cognitive therapy of depression. Nueva York: Guildfor Press. (Trad. cast.: Terapia cognitiva de la depresión. Bilbao: Desclee de Brouwer, 1983).

Becoña, E. (1993). Programa para deixar de fumar. Santiago de Compostela: Servicio de Publicacións da Universidade de Santiago de Compostela.

Becoña, E. (1994a). Tratamiento del tabaquismo. En J.L. Graña (Ed.), Conductas adictivas (pp. 457-493). Madrid: Debate.

Becoña, E. (1994b). Evaluación de la conducta de fumar. En J.L. Graña (Ed.), Conductas adictivas (pp. 373-402). Madrid: Debate.

Becoña, E., Córdoba, R., Díaz-Maroto, J.L., López, V., Jiménez, C., Planchuelo, Ma A., Salvador, T. y Villalbí, J. (2001). Guía de procedimientos para ayudar a los fumadores a dejar de fumar. Adicciones, 13, 211-216.

Becoña, E. y Míguez, M.C. (1995). El cuestionario de evaluación de la recaída/ abstinencia de los cigarrillos: primeros resultados. Revista Española de Drogodependencias, 20, 25-40.

Becoña, E., Palomares, A. y García, M.P. (1994). Tabaco y salud. Guía de prevención y tratamiento del tabaquismo. Madrid: Pirámide.

Becoña, E. y Vázquez, F.L. (1997). Does using relapse prevention increase the efficacy of a program for smoking cessation? An empirical study. Psychological Reports, 83, 1455-1458

Becoña, E. y Vázquez, F.L. (1998a). Self-reported smoking and measurement of expired air carbon monoxide in a clinical treatment. Psychological Reports, 83, 316-318.

Becoña, E. y Vázquez, F.L. (1998b). Tratamiento del tabaquismo. Madrid: Dykinson.

Becoña, E. y Vázquez, F.L. (2001). Effectiveness of personalized written feedback through a mail intervention for smoking cessation: A randomizedcontrolled trial in Spanish smokers. Journal of Consulting and Clinical Psychology, 69, 33-40.

Cohen, S., Kamarck, T.y Mermelstein, R. (1983). A global measure of perceived stress. Journal of Health and Social Behavior, 24, 385-396. 
Condiotte, M.M. y Lichtenstein, E. (1981). Self-efficacy and relapse smoking cessation programs. Journal of Consulting and Clinical Psychology, 49, 648-658.

Connolly, G.N. (1998). Policies regulating cigars. En: National Cancer Institute, Cigars. Health Effects and Trends (pp. 221-249). Bethesda, Maryland: U.S. Department of Health and Human Services. Smoking and Tobacco Control, monograph 9. NIH publication 98-4302.

Fant, R.V. y Henningfield, J.E. (1998). Pharmacology and abuse potencial of cigars. En National Cancer Institute, Cigars. Health Effects and Trends (pp. 160-191). Bethesda, Maryland: U.S. Department of Health and Human Services. Smoking and Tobacco Control, monograph 9. NIH publication 98-4302.

Feit, M.N. (2001). Exposure of adolescent girls to cigar images in women's magazines, 1992-1998. American Journal of Public Health, 91, 286-288.

Gerlach, K.K., Cummings, K.M., Hyland, A., Gilpin, E.A., Johnson, M.D. y Pierce, J.P. (1998). Trends in cigar consumption and smoking prevalence.En: National Cancer Institute, Cigars. Health Effects and Trends (pp.21-52). Bethesda, Maryland: U.S. Department of Health and Human Services. Smoking and Tobacco Control, monograph 9. NIH publication 98-4302.

Gilpin, E.A. y Pierce, J.P. (1999). Cigar smoking in California: 1990-1996. American Journal of Preventive Medicine, $16,195-201$.

Heatherton, T.F., Kozlowski, L.T., Frecker, R.C. y Fagerström, K.O. (1991). The Fagerström Test for Nicotine Dependence: A revision of the Fagerström Tolerance Questionnaire. British Journal of Addiction, 86, 11191127.

Hughes, J.R. y Hatsukami, D.K. (1986). Signs and symptoms of tobacco withdrawal. Archives of General Psychiatry, 43, 289-294

Lando, H.A. (1993). Formal Quit Smoking treatments. En C.T. Orleans y J. Slade. Nicotine Addiction principles and management (pp. 221-244). Nueva York: Oxford University Press

Lichtenstein, E. y Glasgow, R.E. (1992). Smoking cessation: What we learned over the past decade? Journal of Consulting and Clinical Psychology, 60, 804-809.

Llanos, M. (1985). El mundo del tabaco. Madrid: Alhambra.

Míguez, M.C, Vázquez, F.L. y Becoña, E. (2002). Effectiveness of telephone contact as an adjunct to a self-help program for smoking cessation. A randomized controlled trial in Spanish smokers. Addictive Behaviors, 27, 139144.

McConnaughy, E.A., DiClemente, C.C., Prochaska, J.O. y Velicer, W.F. (1989). Stages of change in psychotherapy: A follow-up report. Psychotherapy: Theory, Research, and Practice, 26, 494-503.
McConnaughy, E.A., Prochaska, J.O. y Velicer, W.F. (1983). Stages of change in psychotherapy: Measurement and sample profiles. Psychotherapy: Theory, Research, and Practice, 20, 368-375.

Organización Mundial de la Salud (1992). Trastornos mentales y del comportamiento. Descripciones clínicas y pautas para el diagnóstico (CIE-10). Ginebra: Organización Mundial de la Salud.

Prochaska, J.O., Norcross, J.C. y DiClemente, C.C. (1994). Chaging for good. Nueva York: William Morrow and Cia.

Rigotti, N.A., Lee, J.E. y Wechsler, H. (2000). US college students'use of tobacco. Results of a national survey. JAMA, 284, 699-705

Shanks, T.G. y Burns, D.M. (1998). Disease consequences of cigar smoking. En National Cancer Institute, Cigars. Health Effects and Trends (pp. 105-155). Bethesda, Maryland: U.S. Department of Health and Human Services. Smoking and Tobacco Control, monograph 9. $\mathrm{NIH}$ publication 98-4302.

Shapiro, J.A., Jacobs, F.J. y Thun, M.J. (2000). Cigar smoking in men and risk of death from tobacco-related cancers. Journal of the National Cancer Institute, 92, 333-337.

Slade, J. (1998). Marketing and Promotion of Cigars. En National Cancer Institute (Ed.), Cigars. Health Effects and Trends (pp. 194- 205). Bethesda, Maryland: U.S. Department of Health and Human Services. Smoking and Tobacco Control, monograph 9. NIH publication 984302.

Spielberger, C.D., Gorsuch, R.L. y Lushene, R.E. (1970). STAI. Manual for the State-Trait Anxiety Inventory. Palo Alto, California: Consulting Psychologists Press. (Trad. cast.: Cuestionario de Ansiedad Estado/Rasgo. Madrid: TEA, 1982).

USDHHS (1988). The health consequences of smoking. Nicotine addiction. A report of the Surgeon General. Rockville, MD: U.S. Department of Health and Human Services.

USDHHS (1989). Reducing the health consequences of smoking. 25 years of progress. A report of the Surgeon General. Rockville, MD: U.S. Department of Health and Human Services.

USDHHS (1990). The health benefits of smoking cesssation. A report of the Surgeon General. Rockville, MD: U.S. Department of Health and Human Services.

USDHHS (2000). Treating tobacco use and dependence. Clinical Practice Guideline. Rockville, MD: U.S. Department of Health and Human Services

Velicer, W. F., Prochascha, J. O., Rossi, J. S. y Snow, M. (1992). Assessing outcome in smoking cessation studies. Psychological Bulletin, 111, 23-41. 
